\title{
BMJ Open Effects of home-based mirror therapy and cognitive therapeutic exercise on the improvement of the upper extremity functions in patients with severe hemiparesis after a stroke: a protocol for a pilot randomised clinical trial
}

\author{
Josefa Gonzalez-Santos, ${ }^{1}$ Raul Soto-Camara (1) , ${ }^{1}$ Paula Rodriguez-Fernández, \\ Maria Jimenez-Barrios, ${ }^{1}$ Jeronimo Gonzalez-Bernal, ${ }^{1}$ Carla Collazo-Riobo, ${ }^{1}$
} Maha Jahouh, ${ }^{1}$ Yolanda Bravo-Anguiano, ${ }^{2}$ Jose M Trejo-Gabriel-Galan ${ }^{2}$

To cite: Gonzalez-Santos J, Soto-Camara R, RodriguezFernández $\mathrm{P}$, et al. Effects of home-based mirror therapy and cognitive therapeutic exercise on the improvement of the upper extremity functions in patients with severe hemiparesis after a stroke: a protocol for a pilot randomised clinical trial. BMJ Open 2020;10:e035768. doi:10.1136/ bmjopen-2019-035768

- Prepublication history for this paper is available online. To view these files, please visit the journal online (http://dx.doi. org/10.1136/bmjopen-2019035768).

Received 15 November 2019

Revised 27 June 2020

Accepted 05 August 2020

(C) Author(s) (or their employer(s)) 2020. Re-use permitted under CC BY-NC. No commercial re-use. See rights and permissions. Published by BMJ.

${ }^{1}$ Health Sciences, University of Burgos, Burgos, Spain ${ }^{2}$ Neurology, Burgos University Hospital, Burgos, Spain

Correspondence to Dr Raul Soto-Camara; rscamara@ubu.es

\section{ABSTRACT}

Introduction Neuroplasticity is defined as the capacity of the brain to reorganise new neuronal pathways. Mirror therapy (MT) and cognitive therapeutic exercise (CTE) are two neurorehabilitation techniques based on neuroplasticity and designed to improve the motor functions of the affected upper extremity in patients with severe hemiparesis after a stroke. Home-based interventions are an appropriate alternative to promote independence and autonomy. The objective of this study is to evaluate which of these techniques, MT and CTE, combined with task-oriented training, is more effective in functional recovery and movement patterns of the upper extremities in patients with severe hemiparesis after a stroke.

Methods and analysis This is a home-based, singleblind, controlled, randomised clinical trial with three parallel arms, including 154 patients who had a stroke aged above 18 years. The primary outcome will be the functionality of the affected upper extremity measured using the Fugl-Meyer Assessment. Secondary variables will include cognitive performance, emotional state, quality of life and activities of daily living. During 6 weeks, one of the intervention groups will receive a treatment based on MT and the other one on CTE, both combined with task-oriented training. No additional interventions will be provided to the control group. To assess the progress of patients who had a stroke in the subacute phase, all variables will be evaluated at different visits: initial (just before starting treatment and 4 weeks post-stroke), post-intervention (6 weeks after initial) and follow-up (6 months).

Ethics and dissemination This protocol has been approved by the Institutional Review Board (CEIm2.134/2.019) and registered at ClinicalTrials.gov (NCT04163666). The results will be disseminated through open-access peer-reviewed journals, conference presentation, broadcast media and a presentation to stakeholders. These study results will provide relevant and novel information on effective neurorehabilitation
Strengths and limitations of this study

- This study will use stroke-related neurorehabilitation techniques, which would enable an easy athome application to the patient.

- The sample size will provide greater confidence and credibility regarding the benefits of these neurorehabilitation approaches and help in understanding the relevant aspects to conduct future studies.

- Because of the nature of the intervention, the participants will not be blinded; however, the researchers who perform the measurements and statistical analysis will be blinded

strategies and improve the quality of intervention programmes aimed at patients after a stroke. Trial registration number ClinicalTrials.gov (NCT04163666).

\section{INTRODUCTION}

The WHO defines stroke as a clinical syndrome of vascular origin, characterised by the rapid development of signs of focal and, on occasions, overall neurological deficit, lasting for more than 24 hours. ${ }^{1}$

Stroke requires urgent neurological assistance and is the principal cause of physical disability among adults. ${ }^{2}$ It has been estimated that one-third of stroke-affected people remain dependent on others for care because they have an affectation in performing activities in any domain of life, including basic activities of daily living (BADL) and instrumental activities of daily living (IADL), ${ }^{3}$ because of sensory-based motor disorders; perception disorders; language and communication 
problems; and emotional, psychological, and behavioural disorders. ${ }^{45}$

Weakness (hemiparesis) or paralysis (hemiplegia) on the opposite side of the injured side is the first sequela of stroke in $80 \%$ of the patients. ${ }^{46}$ Severe hemiparesis of the upper extremities, classified according to the Brunnstrom Scale between stages II and IV or II and V depending on the author who considers it, is the most frequent cause of functional disability. ${ }^{7}$ It is defined as the modification in the ability to perform a normal level of muscular strength, including altered sensitivity, weakness, motor control and spasticity. It limits the performance of activities of daily living (ADL), significantly affecting the quality of life of patients who had a stroke. ${ }^{8-11}$ Studies have shown that functional deficits after a stroke are determined by different factors, including the structural extent of the damage and the level of cortical stimulation during the active or passive movement of the affected extremity. This fact must be considered in patients with severe hemiparesis, as first, the motor impairment they present prevents or limits the performance of functionally relevant activities of the affected extremity; and second, severe hemiparesis is commonly accompanied by sensory deficits. Therefore, despite increasing the use of the affected extremity to perform activities in traditional therapies, activation at the cortical level remains very limited. ${ }^{8-11}$

In the stroke rehabilitation process, special attention must be provided for the recovery of functional and motor activities of the affected upper extremity, needed for performing ADL. ${ }^{9}$ It typically commences with spontaneous recovery during the first month, followed by learning-assisted recovery during the next 6 months and slow recovery during the subsequent months. This can be achieved as recovery is even possible in the most chronic phases because of cerebral plasticity and motor-related sensory learning. ${ }^{12-14}$ Most studies have shown the importance of both patient-centred interventions and the need to combine different techniques, thereby not centring the treatment on a single modality. ${ }^{1315}$

Home-based therapy programmes for recovery after a stroke significantly improve independence and participation in ADL. These programmes reduce long-term dependency ${ }^{1617}$ and are at least as effective as hospital interventions. ${ }^{18}$ Despite the limited number of studies reporting on specific home-based therapy programmes for the functional recovery of the upper extremity following a stroke, ${ }^{16} 19$ people who receive interventions for improving the functionality and reducing deterioration in the upper extremities have been shown to be more independent and more likely to maintain these skills in the long term if they receive this kind of therapy service. ${ }^{20}$ At home, patients are forced to face the real challenges of daily life; therefore, in addition to improving functional outcome and satisfaction, ${ }^{21} 22$ this type of intervention reduces depression ${ }^{23}$ and encourages motivation and generalisation of learning. ${ }^{24} 25$

This study is designed to create a home-based therapy programme for the functional recovery of the upper extremities using mirror therapy (MT) or cognitive therapeutic exercise (CTE) in combination with task-oriented training and to verify which of these two techniques is more effective in functional recovery and movement patterns of the upper extremities in patients with severe hemiparesis after a stroke.

\section{Mirror therapy}

The objective of MT, developed by Ramachandran and Rogers-Ramachandran, ${ }^{26}$ is to improve motor functions of the affected upper extremities. In this therapy, the patient is guided such that the attention is centred on the reflection of the healthy upper extremity in a mirror as if it was the affected one. ${ }^{6}$ The observation of normal movements in the mirror provides positive visual feedback and increases the flow of proprioceptive information and activates the neurons and the premotor cortex, thus improving the functionality of the affected upper extremity. ${ }^{27-29}$

Numerous studies have shown the benefits of MT in the rehabilitation of motor functions of the upper extremity, the performance of ADL and pain thresholds of patients who had a stroke. ${ }^{27-34}$ However, Oliveira-Castro et $a l^{35}$ did not observe considerable improvements in self-care because of different limitations such as the short duration of the programme, small sample size and lack of randomisation.

Factors such as age, cognitive capability and fingerrelated motor functioning of the affected or dominant hand affect the degree of effectiveness of MT in patients with hemiparesis. ${ }^{6}$ Therefore, the majority of studies have agreed on the need for further research in this area. ${ }^{27-35}$

\section{Cognitive therapeutic exercise}

The objective of CTE or the Perfetti method, a cognitive rehabilitation modality, is to achieve motor functions using afferent information of a proprioceptive and/or tactile type. The proprioceptive information received is associated with the recognition of the direction, distance and shapes through the body. The information associated with touch refers to the recognition of characteristics that are deduced by touching the object. The problem raised while perceiving the information can only be solved if the nature, intensity and characteristics of the contact made are recognised. Therefore, the exercises are based on what the patient needs to recognise in an object: surface, pressure, friction resistance or weight. ${ }^{36}$

The patient is expected to bring attention to parts or specific characteristics of the body while solving a cognitive problem that is described by the therapist. The problem is presented to the patient as the preparation of a perceptive hypothesis in such a way that the patient consciously perceives the upper extremity in a manner that favours the re-learning of a motor action that has been lost by the nervous system. ${ }^{37}$

CTE has been effective in improving muscle strength, tactile sensorial discrimination and kinesthetics, the functionality of the upper hemiplegic or hemiparetic 
extremities, and the quality of life of patients after a stroke. ${ }^{38-41}$

However, despite the fact that it is reported as one of the most complete and effective methods for the rehabilitation of neurological deficit in the upper extremities, ${ }^{38}$ new studies with a larger sample size are warranted, which could define aspects such as time and materials required for their implementation. ${ }^{37-39}$

\section{Task-oriented training}

Task-oriented training is an effective method to promote and stimulate motor skills and neuronal plasticity in patients with injuries to the central nervous system (CNS). It included the screening of the patient to develop personal strategies for performing useful functional movements in different contexts and situations. ${ }^{41}$ The paretic hemibody is trained under suitable conditions using specific, repetitive and intensive tasks, which are crucial for the patient. ${ }^{4-44}$ By actively participating in the rehabilitation process and intervening in the search and discovery of strategies, personal functional independence is improved, and the adaptation of the patient to the environment is assisted. ${ }^{41}$ This rehabilitation method uses five strategies to teach and motivate the patient, who must adapt to the requirements of each task: verbal instructions, visual demonstrations, manual guide, reinforcement and positive feedback, and its repetitive practice in different contexts. ${ }^{41}$

Despite scarce scientific evidence in this field in the literature, it has been observed that therapies with taskoriented functions facilitate greater functional recovery of the upper extremities. Therefore, a therapeutic approach based on task-oriented training that stimulates the potential neuroplasticity of CNS combined with techniques such as MT and CTE is of greater use in improving the motor function than the approaches based on a specific or concrete technique. ${ }^{41434546}$

Thus, a comparative study between MT and CTE has been planned. Although moderate-quality evidence exists to suggest that both techniques are effective in neurorehabilitation, their comparative effectiveness, or the one that yields better results and maintenance over time is yet to be confirmed. ${ }^{47}$ Therefore, the objective of this study is to evaluate which of these techniques combined with task-oriented training is more effective in functional recovery and movement patterns of the upper extremities in patients with severe hemiparesis after a stroke.

\section{METHODS AND ANALYSIS \\ Design and setting}

This is a single-blinded, controlled, randomised clinical trial with three parallel arms (control, intervention 1 and intervention 2). Participants will be enrolled from 1 January 2020 until 18 months. At the first visit, just 1 month after having suffered from a stroke, patients will be determined to meet the inclusion criteria, and the initial evaluation will be performed for patients who meet the criteria. A post-intervention visit after 6 weeks will be conducted, and a follow-up visit will be conducted 6 months after a stroke to evaluate the progress of patients in the subacute phase of recovery.

\section{Study population}

The participants will be recruited at the point of discharge from the Neurological Service and Stroke Unit of the Burgos University Hospital (Spain) by means of consecutive sampling. This is the only third-level health centre for the referral of patients who had a stroke in the region. All evaluation and follow-up visits and the development of the interventions will be carried out in the patient's home.

All participants meeting the following inclusion criteria would be included in the study: patients of both sexes, those aged 18 years or above, those with a diagnosis of residual hemiparesis because of ischaemic or haemorrhagic stroke, those whose movements of the affected upper extremities are classified between stages II and IV on the Brunnstrom Scale, ${ }^{48}$ and those with a score on the Montreal Cognitive Assessment (MoCA) Scale $^{49}$ equal to or above 26. All participants will be required to sign an informed consent form. Participants presenting hemineglect, Wernicke's aphasia, mixed aphasia and/or visual deficits (homonymous hemianopsia) will be excluded from the study, considering the diagnostic information provided by the clinical assessment of neurologists.

\section{Patient and public involvement}

The patients and the public will participate in the study design so that time and spaces necessary for the homebased intervention could be adapted according to their availability. Moreover, they will be part of the data collection process and will be informed of the results obtained. Participants may suggest changes related to the frequency and intensity of the sessions. The results will be disseminated through communications, including media, healthcare institutions, patients' associations for which collaborative agreements are in place and during meetings organised for sharing information with the participants of the study.

\section{Estimation of the sample size}

The sample size has been estimated on the basis of the potential modification of the main variable, that is, the functionality of the affected upper extremity. Given alpha and beta risks of 0.05 and 0.20 , respectively, in bilateral contrast, 110 participants ( 55 per group) will be required to detect a minimum difference of 0.50 in the functionality of the affected upper extremity using the Fugl-Meyer Assessment (FMA) between the two groups. Additional 44 individuals will be needed for calculating the size of the spontaneous improvement group, which is estimated to occur in $20 \%$ of the cases. ${ }^{48}$ A predicted dropout rate of $10 \%$ during follow-up has been considered. 


\section{Randomisation}

Participants will be randomly assigned either to a control group (CG) or an intervention group (IG). An assignation sequence in masking clusters at a ratio of 1:1:1 will be centrally generated by an independent researcher using the Epidat 4.2 program before the inclusion of the participants.

\section{Intervention}

The standard rehabilitation treatment for stroke will be used for all study participants. Participants included in the CG will not receive any additional treatment or therapy.

IG-1 will receive an additional treatment of MT with task-oriented training, whereas IG-2 will receive CTE with task-oriented training. In both groups, the interventions will begin at the participant's home during a period of 6 weeks (30 days), including 60-min sessions, $30 \mathrm{~min}$ of which will be for MT or CTE, and the remaining $30 \mathrm{~min}$ will be for task-oriented training.

\section{Task-oriented training}

Task-oriented training will be presented sequentially in a structured manner along with their specific characteristics, which would vary from one session to another to facilitate in their application in daily life. The complex activities will be divided into simpler tasks for easy learning. Verbal instructions and/or presentations will be provided, gradually decreasing the support. Every day of the week will be dedicated to a different BADL among the following: diet (preparing and organising food, as well as time taken to eat), clothing (upper and lower extremities), and personal hygiene (brushing teeth, combing hair, and shaving or applying makeup). There will be short resting periods, and the difficulty of tasks will be progressively increased to assist carry over. The last week will be dedicated to tasks and BADL that the patient requests and can be performed at home. ${ }^{50}$

\section{Mirror therapy}

In the MT, the patient will be seated on an ordinary chair, with their forearms resting on a table. A mirror will be placed between both the arms at a right angle to the torso. The affected extremity must be placed behind the mirror, in a comfortable position, in such a way that the patient cannot see it. The healthy extremity must be placed in a position as similar as possible to the affected extremity so that it completely reflects in the mirror with no distortion of the image. Any object or symbol that can identify the healthy extremity must be removed or covered up (figure 1).

MT can be used in three different modes. In the first mode, the patient tries to imitate the movement of the healthy hand with the affected hand in a synchronised way. In the second mode, the patient only imagines that the reflected movement of the healthy hand is being performed by his affected hand. In the third mode, the therapist assists the patient's affected hand to imitate the movement performed by the healthy hand. Considering

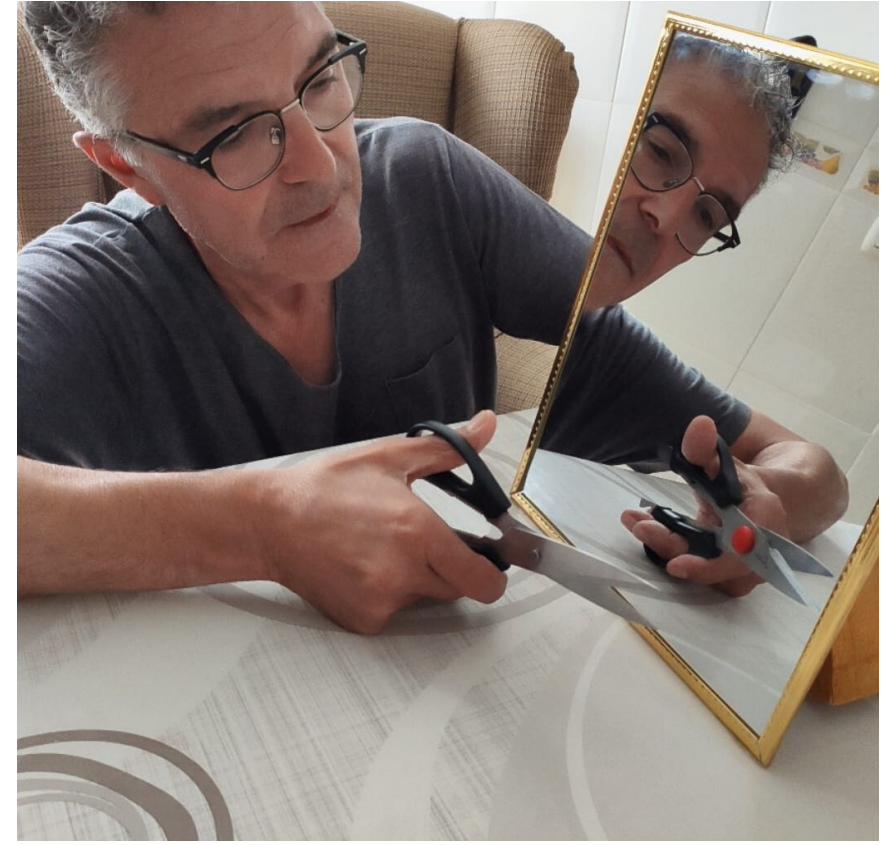

Figure 1 In the intervention set up for mirror therapy, the participant looks at the reflection of the unaffected hand in the mirror as if it was the affected hand.

these modes, first simple exercises without objects were performed with the healthy hand while imagining the same movement with the affected hand; then, the same movements are performed by the affected hand with the help of the therapist. ${ }^{5152}$

Simple movements without any objects will be performed during the first 10 sessions, and then, simple movements using objects will be performed in the following 10 sessions. Moreover, movements of greater complexity with objects will be included in the last 10 sessions. To be more specific, the patient will complete movements with a healthy hand throughout the first 3 groups of 10 sessions and will try to imagine them with the affected hand (mode). Those movements will be assisted by a therapist in the three subsequent sessions (mode), whereas the patient will try to imitate the movements of the healthy hand by the affected hand throughout the last four sessions (mode). All the exercises will have to be completed slowly and repeated at least 15 times. The activities and their levels of complexity will be adjusted as per the limitations and capabilities of each patient. ${ }^{5152}$

\section{Cognitive therapeutic exercise}

The CTEs are classified according to multiple criteria in three different modalities: first, second and third grades. All patients will begin performing the first-grade exercises until they regain the ability to control the reaction to stretching to graduate intensity, time and spatiality. Once the patient can successfully perform the first-degree exercises, control the reactions to stretching in a sufficiently automated way, and frequently perform selective movements of the fingers and other body segments, the second-degree exercises are performed..$^{39} 53$ 
Finally, third-degree exercises are performed through which the patient learns to adapt movements to the proposed perceptual hypothesis after the patient has managed to automate the control of abnormal motor behaviours with the second-degree exercises. The criteria that must be considered to establish correct programming of the exercises depend on the configuration of the trajectories that are requested from the patient and on the intensity of the contractions that must be activated in the segments that execute them. ${ }^{36}$

In the first-degree modality, the aim is to control the exaggerated reaction to stretching (commonly known as spasticity) and overcome the sensitivity deficit, where the therapist performs the patient's movements. These exercises are not entirely considered passive as they require the patient's attention at all times. Moreover, in the seconddegree modality, the objective is to control abnormal irradiation (involuntary activation of muscle groups). In this case, the movements are performed by the patient with the minimum necessary help from the therapist. These exercises include tactile, kinesthetic, weight, grip and friction input. This modality will be introduced in combination with the first-degree exercises once the patient has acquired a certain degree of control over the recruitment of motor units. Finally, in the third-grade modality, the control of voluntary movements and the decisions on their fragmentation, variability and adaptation will be emphasised, with the aim of achieving perfect automation of movements where the patient is totally active and does not need any type of help from the therapist. The information is received through the same method as in the previous modality. ${ }^{36354}$

For its application, the CTE always proposes each exercise in the same structure such that first, a cognitive problem is proposed, then a perceptual hypothesis (feedforward) is activated to solve the problem and finally, a comparison process occurs between the previous and sensory information (feedback) that the patient receives during the therapeutic exercise. ${ }^{53}$ Before starting the activity, the patient is shown different materials or subsidies using which the exercise is performed. During this time, the patient must recognise the possible strategies that help in solving the problem by collecting information through his body. ${ }^{53}$ Therefore, the elements present in any exercise of the present technique are the cognitive problem, perceptual hypothesis and verification of that hypothesis.

For example, the therapist shows the patient three two-dimensional figures (triangle, circle and square), allowing the patient to observe and touch them. Then, the patient closes his eyes and touches the figures with his fingertips (guided or not by the professional), and with the information received through the body, the patient should recognise the figure.

\section{Procedure}

An initial evaluation visit will be completed in the 4th week after a stroke, and a post-intervention visit at week

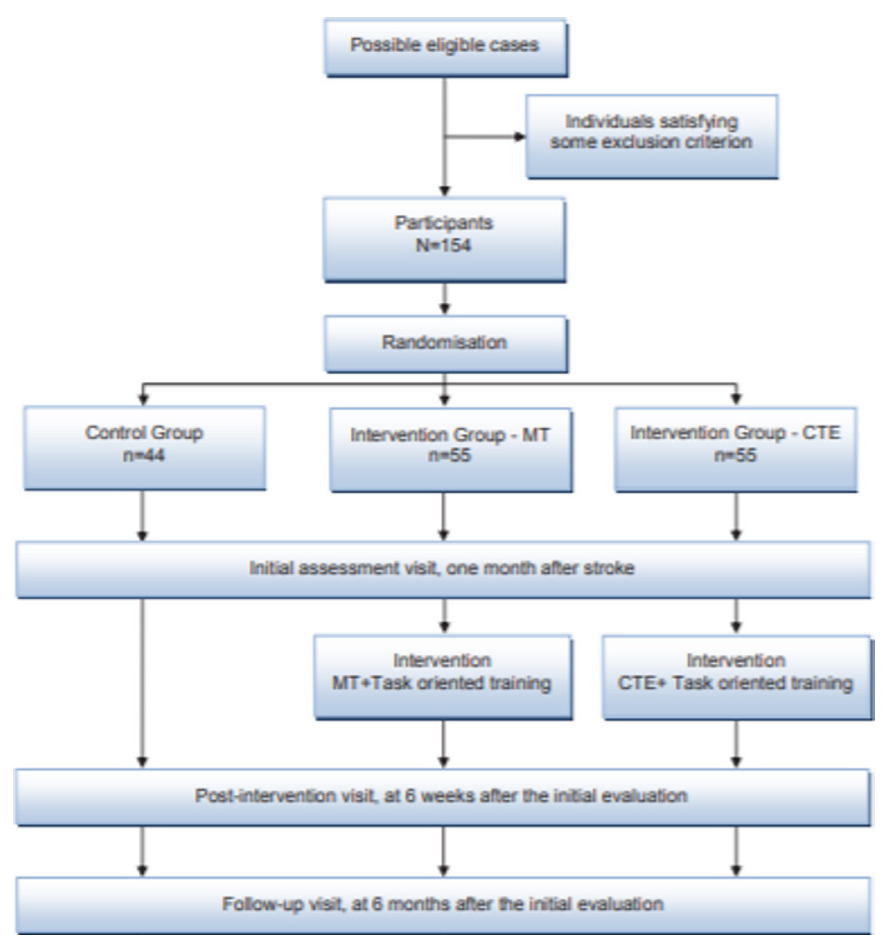

Figure 2 Study flow chart. CTE, cognitive therapeutic exercise; MT, mirror therapy;

6 will be performed. A follow-up visit will occur 6 months post-stroke to evaluate the progress of patients who had a stroke in the subacute phase of recovery. Both IG and CG will undergo all the evaluations. Their state will be assessed using different instruments and questionnaires in all visits.

All participants included in the IG will receive 30 treatment sessions, distributed between the initial evaluation and the first follow-up visit (figure 2).

\section{Primary and secondary endpoints}

The primary outcome will be an improvement in the functionality of the affected upper extremity. The secondary variables will include cognitive performance, emotional state, quality of life and performance of ADL. The primary and secondary endpoints will be evaluated at 6 months post-stroke through the use of questionnaires, previously validated for the Spanish population.

\section{The functionality of the affected upper extremity}

To perform a thorough assessment, different motor deficit components of the affected upper extremity will be evaluated using the FMA, which will be combined with four other scales:

- The FMA,$^{55}$ which assesses motor functioning, passive articular mobility, articular pain, coordination and balance.

- The Action Research Arm Test ${ }^{56}$ which measures the functionality of the upper extremity.

- The Motor Activity Log $30{ }^{56}$ which assesses the quantity (amount subscale -AS) and quality (how well subscale - HW) of the use of hand and arms during the performance of ADL. 
- The Block and Box Test, ${ }^{57}$ which assesses the manipulative value of the hand.

- The Modified Ashworth Scale, ${ }^{58}$ which measures the spasticity of all the movements of the different joints of the upper extremity: shoulder (flexion, extension, abduction, adduction, and internal and external rotation); elbow (flexion, extension, pronation and supination); wrist (flexion, extension, and ulnar and radial deviation); distal and proximal metacarpophalangeal and interphalangeal (flexion and extension); the second to fifth fingers (abduction and adduction) and thumb (flexion, extension, abduction and adduction).

\section{Cognitive performance}

The cognitive performance will be evaluated using the MoCA scale, ${ }^{49}$ distributed into seven different cognitive domains: visuospatial executive capacity; denomination; language attention; abstraction; learning; and deferred learning, recall and orientation.

\section{Emotional state and quality of life}

Emotional state and quality of life will be evaluated using the Escala de Calidad de Vida para el Ictus (Scale of PostStroke Quality of Life) ${ }^{59}$ which comprises 38 items grouped into eight domains: physical state, communication, cognition, emotions, feelings, BADL, common ADL and socio-family functioning.

\section{Performance of ADL}

BADL will be evaluated using the Functional Independence Measure and its extension, the Functional Assessment Measure, designed specifically for patients with cerebral damage. ${ }^{60}$ The 30 items in this instrument are used to assess the self-care tasks inherent to ADL, motor, cognitive, and behavioural functions and communicative and functional behaviour in the community.

The IADL will be evaluated using the Lawton-Brody Index. ${ }^{61}$ It assesses the capability to develop tasks involving the handling of everyday utensils and day-to-day social activities, including telephone use, shopping, preparing meals, household work, washing clothes, transport use, responsibility for medication administration and management of economic affairs.

\section{Other measures/covariables}

The following variables will be evaluated to control the possible predictive or confusion factors: age, sex, type of stroke, affected cerebral hemisphere and stroke severity, quantified by the National Institute Health Stroke Scale. ${ }^{62}$

\section{Data collection, data management and follow-up procedure}

To maintain the quality of data collection, the initial evaluation, follow-up and intervention visits will be conducted by properly trained and accredited therapists, who would follow the standardised methodological criteria mentioned in the data collection manual. Therapists conducting initial and follow-up visits will be different from those who provide different rehabilitation techniques. Each participant will be assigned a unique intervention code for this study. All the assessments would be entered into a safe and secure data collection notebook. A database would be created using the SPSS software V.25.00, accessible only to the research team members and the ones involved in the statistical analysis of the study. A researcher on a weekly basis will monitor the inclusion of new patients, debugging, testing, checking of databases and adaption of protocol procedures. Double entry of data will be performed to maintain a low error rate.

\section{Blinding strategy}

This is a single-blinded study. Because of the nature of the intervention, the participants and people responsible for using the MT or CTE, both combined with taskoriented training, to IG cannot be blinded. However, the person taking the measurements during the follow-up visit and the researcher analysing the data statistically will be blinded with respect to the group to which the participants belong. In addition, clear instructions will be provided to the participants of not revealing the group to which they have been assigned during the assessment visits.

\section{Statistical analysis}

General analysis

The results of the main and the secondary variables will be analysed using intention-to-treat analysis to control the effects of non-random abandonment. The mean and SD will be used for the description of the quantitative variables or frequency distribution and percentages for categorical variables. The normality of the variables will be assessed using the Kolmogorov-Smirnov test. In cases where the normal distribution cannot be assumed, median, IQR and the corresponding non-parametric test will be used. The association between independent categorical variables will be analysed using $\chi^{2}$ test or Fisher's exact test. The means between the two groups will be compared using the Student's t-test or the Mann-Whitney U-test. Pearson's or Spearman's correlation coefficients will be calculated to analyse the relationship between quantitative variables. A $p$ value of $<0.05$ was considered statistically significant. The statistical analysis will be performed using the SPSS software V.25.0.

\section{Analysis of the effects of the intervention on primary and} secondary outcomes

To analyse the changes at 6 weeks and 6 months from baseline in the primary (functionality of the affected upper extremity) and secondary outcomes within the same group, the Student's t-test for paired data or Wilcoxon test will be used.

The effects of the intervention will be analysed by comparing the changes in the functionality of the affected upper extremity between groups using the analysis of covariance of change score, with the baseline as covariate and by adjusting for possible confounders. The effects of 
the intervention during follow-up will be studied using an analysis of the variance of repeated measures.

\section{Analysis by subgroups}

The effects of the intervention can be influenced by age, sex, type of stroke, affected cerebral hemisphere and stroke severity. The same analysis described above will be performed for each of the subgroups.

\section{Secondary analysis}

A multivariate multiple regression analysis will be performed to identify the variables that greatly influence the changes in the functionality of the affected upper extremity and the secondary variables analysed.

\section{ETHICS AND DISSEMINATION Ethical considerations}

The study has been approved by the Clinical Research Ethics Committee of the Health Area of Burgos and Soria (Spain) in June 2019 (CEIm 2.134/2.019). A Standard Protocol Items: Recommendations for Interventional Trials declaration checklist is available for this protocol.

In accordance with the Helsinki Declaration, prior informed consent will be obtained from the individuals who voluntarily decide to participate. They will be informed of the objectives, risks and benefits of the assessments, as well as the interventions that will be used. None of the activities imply additional risks to the life of the participants. At all times, the confidentiality of the data will be guaranteed in accordance with the current legislation.

\section{Dissemination plan}

The dissemination of results will be as per the recommendations mentioned in the Consolidated Standards of Reporting Trials declaration. This study will be published in open access peer-reviewed scientific journals, so as to acquire the highest possible visibility for the study. At least two publications are expected to be completed, one of the results of the primary outcome (functionality of the upper extremity) and the other on the secondary results. The results will be disseminated through communications, including media and social networks, as well as at international and national scientific conferences and seminars. Similarly, a doctoral thesis based on the content of this project will be developed.

\section{DISCUSSION}

At present, a number of rehabilitative methods exist based on the principle of cerebral plasticity. Among these, MT and CTE promote the recovery of the affected upper extremity.

Various clinical trials have demonstrated that MT is an effective treatment for the recovery of the upper extremity in patients who had a subacute stroke. ${ }^{27-34}$ Vandana et at ${ }^{48}$ evaluated the effects of MT on motor recovery of the upper extremities, spasticity and hand-related functionality of patients who had a subacute stroke and severe hemiparesis (stages II-IV of the Brunnstrom Scale). Moreover, a greater improvement in the scores of the Brunnstrom stages for the hand and upper extremity was observed in the MT group than the conventional therapy group. Arya $e t a t^{63}$ evaluated a specific task-based neurorehabilitation therapy among patients who had a subacute stroke with severe hemiparesis (stages II-V of the Brunnstrom Scale) and showed that there were greater improvements in performing activities with this method than with any other conventional method. Lisalde-Rodríguez et $a b^{64}$ demonstrated that MT combined with conventional therapy was effective in improving the motor function of the upper paretic extremity but not the overall functionality of the patient. In another study, MT combined with task-oriented training within the same treatment session showed statistically significant results in the functionality of the upper extremity and the performance of ADL in the patient. ${ }^{43} \mathrm{Kim}$ et $a l^{12}$ involving 25 patients who had a stroke confirmed the effectiveness of an intervention with MT combined with task-oriented training in comparison to conventional therapy. MT combined with task-oriented training is the most effective method in the rehabilitation of the affected upper extremity functionality and performance of ADL.

Despite having demonstrated the effectiveness of CTE in executing simple motor activities such as cylindrical grip, it lacks evidence on its repercussions on the recovery of distal functions of greater complexity among patients with the affected upper extremities following a stroke. ${ }^{38}$

Although this study relates both the treatment techniques, it presents significant limitations such as lack of specificity on the level of deficit affecting the upper extremity, sample size and the control groups. It is necessary to determine the correct execution and description of activities, processes and exercises for their development and, similarly, to validate future investigations on a scientific basis. No studies to date have demonstrated which out of the two techniques is the most effective in achieving functional recovery of the affected upper extremity.

This study will provide novel and useful results for the development of post-stroke rehabilitation strategies. The intervention may provide implications for the preparation of evidence-based recommendations, practical clinical guidelines and continuous quality improvement programmes for patients with severe hemiparesis after a stroke.

The relevant information will be obtained about the functionality of the upper extremity of patients with severe hemiparesis after the practice of a more intensive therapy that combines two types of neurorehabilitation approaches.

The sample size of this study helps in understanding the relevant aspects of conducting future studies, and it will also provide greater confidence and credibility regarding the benefits of these neurorehabilitation approaches. 
Until now, the effectiveness of both techniques is known; however, this study will facilitate the professional to select the technique that should be implemented in his rehabilitation to achieve the best possible results.

Lastly, the fact that it is a home-based intervention that combines MT and CTE with task-oriented training will help us to discover if these factors influence the transfer of the movement patterns learnt during the different interventions to the execution of ADL.

Contributors JGS, PR-F, MJ-B, CC-R and MJ devised the design of the study. JG-B, PR-F, MJ-B and RS-C prepared and developed the protocol study. JGS, JG-B and RS-C provided methodological assistance and statistical assessment. YB-A and JMT-G-G provided a critical review of the paper. All the authors have read and accepted the final version of the protocol.

Funding The project leading to these results has received funding from "La Caixa" Foundation, under agreement LCF/PR/PR18/51130007>.

Competing interests None declared.

Patient and public involvement Patients and/or the public were not involved in the design, or conduct, or reporting, or dissemination plans of this research.

Patient consent for publication Not required.

Provenance and peer review Not commissioned; externally peer reviewed.

Open access This is an open access article distributed in accordance with the Creative Commons Attribution Non Commercial (CC BY-NC 4.0) license, which permits others to distribute, remix, adapt, build upon this work non-commercially, and license their derivative works on different terms, provided the original work is properly cited, appropriate credit is given, any changes made indicated, and the use is non-commercial. See: http://creativecommons.org/licenses/by-nc/4.0/.

\section{ORCID iD}

Raul Soto-Camara http://orcid.org/0000-0002-9072-0364

\section{REFERENCES}

1 Hatano S. Experience from a multicentre stroke register: a preliminary report. Bull World Health Organ 1976;54:541-53.

2 Organización Mundial de la Salud. Enfermedades cardiovasculares, 2017. Available: https://www.who.int/es/news-room/fact-sheets/ detail/cardiovascular-diseases-(cvds)

3 Legg LA, Drummond AE, Langhorne P. Occupational therapy for patients with problems in activities of daily living after stroke. Cochrane Database Syst Rev 2006;4:CD003585.

4 Polonio López B, Romero Ayuso DM. Terapia Ocupacional aplicada al daño cerebral adquirido. Madrid: Editorial Médica Panamericana SA, 2010.

5 Ministerio de Sanidad y Política Social. Guía de Práctica Clínica para El manejo de pacientes Con ictus en Atención Primaria. Madrid, 2009.

6 Brunetti M, Morkisch N, Fritzsch C, et al. Potential determinants of efficacy of mirror therapy in stroke patients-A pilot study. Restor Neurol Neurosci 2015;33:421-34.

7 Dohle C, Püllen J, Nakaten A, et al. Mirror therapy promotes recovery from severe hemiparesis: a randomized controlled trial. Neurorehabil Neural Repair 2009;23:209-17.

8 Fernández-Gómez E, Sánchez-Cabeza A. [Motor imagery: a systematic review of its effectiveness in the rehabilitation of the upper limb following a stroke]. Rev Neurol 2018;66:137-46.

9 Wist S, Clivaz J, Sattelmayer M. Muscle strengthening for hemiparesis after stroke: a meta-analysis. Ann Phys Rehabil Med 2016;59:114-24.

10 Hara T, Abo M, Kakita K, et al. Does a combined intervention program of repetitive transcranial magnetic stimulation and intensive occupational therapy affect cognitive function in patients with post-stroke upper limb hemiparesis? Neural Regen Res 2016;11:1932-9

11 Kakuda W, Abo M, Sasanuma J, et al. Combination protocol of lowfrequency rTMS and intensive occupational therapy for post-stroke upper limb hemiparesis: a 6-year experience of more than 1700 Japanese patients. Trans/ Stroke Res 2016;7:172-9.
$12 \mathrm{Kim} \mathrm{K}$, Lee S, Kim D, et al. Effects of mirror therapy combined with motor tasks on upper extremity function and activities daily living of stroke patients. J Phys Ther Sci 2016;28:483-7.

13 Schaechter JD. Motor rehabilitation and brain plasticity after hemiparetic stroke. Prog Neurobiol 2004;73:61-72.

14 Murie-Fernández M, Irimia P, Martínez-Vila E, et al. Neurorehabilitation after stroke. Neurología 2010;25:189-96.

15 Flórez García MT. I interventions to improve motor function in stroke patients. Rehabilitación 2000;34:423-37.

16 Aziz NA, Leonardi-Bee J, Phillips M, et al. Therapy-based rehabilitation services for patients living at home more than one year after stroke. Cochrane Database Syst Rev 2008;2:CD005952.

17 Langhorne P, Baylan S, Early Supported Discharge Trialists. Early supported discharge services for people with acute stroke. Cochrane Database Syst Rev 2017;7:CD000443.

18 Roderick P, Low J, Day R, et al. Stroke rehabilitation after hospital discharge: a randomized trial comparing domiciliary and day-hospital care. Age Ageing 2001;30:303-10.

19 Coupar F, Pollock A, Legg LA, et al. Home-based therapy programmes for upper limb functional recovery following stroke. Cochrane Database Syst Rev 2012;5:CD006755.

20 Outpatient Service Trialists. Therapy-based rehabilitation services for stroke patients at home. Cochrane Database Syst Rev 2003;1:CD002925.

21 Minelli C, Gondim FAA, Barreira AA, et al. Rehabilitation of the upper extremity and basic activities of daily living in the first month after ischemic stroke: an international cohort comparison study. Neurol Int 2009;1:e4.

22 Gilbertson L, Langhorne P, Walker A, et al. Domiciliary occupational therapy for patients with stroke discharged from Hospital: randomised controlled trial. BMJ 2000;320:603-6.

23 Chaiyawat $\mathrm{P}$, Kulkantrakorn K, Sritipsukho P. Effectiveness of home rehabilitation for ischemic stroke. Neurol Int 2009;1:36-40.

24 Björkdahl A, Nilsson AL, Grimby G, et al. Does a short period of rehabilitation in the home setting facilitate functioning after stroke? A randomized controlled trial. Clin Rehabil 2006;20:1038-49.

25 Thorsén A-M, Holmqvist LW, de Pedro-Cuesta J, et al. A randomized controlled trial of early supported discharge and continued rehabilitation at home after stroke: five-year follow-up of patient outcome. Stroke 2005;36:297-302.

26 Radajewska A, Opara JA, Kucio C, et al. The effects of mirror therapy on arm and hand function in subacute stroke in patients. Int $J$ Rehabil Res 2013;36:268-74.

27 Paik Y-R, Kim S-K, Lee J-S, et al. Simple and task-oriented mirror therapy for upper extremity function in stroke patients: a pilot study. Hong Kong J Occup Th 2014;24:6-12.

28 SFM T, Fong KNK. Systematic review on the effectiveness of mirror therapy in training upper limb hemiparesis after stroke. Hong Kong $J$ Occup Th 2012;22:84-95.

29 Novaes MM, Palhano-Fontes F, Peres A, et al. Neurofunctional changes after a single mirror therapy intervention in chronic ischemic stroke. Int J Neurosci 2018;128:966-74.

30 Fukumura K, Sugawara K, Tanabe S, et al. Influence of mirror therapy on human motor cortex. Int J Neurosci 2007;117:1039-48.

31 Bai Z, Zhang J, Zhang Z, et al. Comparison between movementbased and task-based mirror therapies on improving upper limb functions in patients with stroke: a pilot randomized controlled trial. Front Neurol 2019;10:288.

32 Zeng W, Guo Y, Wu G, et al. Mirror therapy for motor function of the upper extremity in patients with stroke: a meta-analysis. J Rehabil Med 2018;50:8-15.

33 Park J-Y, Chang M, Kim K-M, et al. The effect of mirror therapy on upper-extremity function and activities of daily living in stroke patients. J Phys Ther Sci 2015;27:1681-3.

34 Costa DS V, Cunha da Silveira JC, Albuquerque Clementino TC, et al Effects of mirror therapy on the motor and functional recovery of post-stroke paretic upper limbs: a systematic review. Fisioter Pesqui 2016;23:431-8.

35 Oliveira e Castro P, Martins M, Couto G, et al. Mirror therapy and self-care autonomy after stroke: an intervention program. Revista de Enfermagem Referência 2018;4:95-106.

36 Perfetti C, Ghedina R, Jiménez Hernández D. El ejercicio terapeútico cognoscitivo para La reeducación motora del hemipléjico Adulto. Edika Med: Barcelona, 1999.

37 Domínguez Ferraz D, Da Silva-Ribeiro NM, Matos-Pinheiro D I, et al. Eficacia del método Perfetti en El tratamiento de secuelas del accidente cerebrovascular: Una revisión sistemática. Cuest Fisioter 2014;43:196-205.

38 Díaz Castro WM, Rodríguez López YC. Método Perfetti como Estrategia Terapéutica en La Rehabilitación de Pacientes Con 
Enfermedad cerebrovascular: Revisión Bibliográfica. Mov Cient 2019:13:65-70.

39 Sallés L, Martín-Casas P, Gironès X, et al. A neurocognitive approach for recovering upper extremity movement following subacute stroke: a randomized controlled pilot study. J Phys Ther Sci 2017;29:665-72.

40 Lee S, Bae S, Jeon D, et al. The effects of cognitive exercise therapy on chronic stroke patients' upper limb functions, activities of daily living and quality of life. J Phys Ther Sci 2015;27:2787-91.

41 Cano de la Cuerda R, Molero Sánchez A, Carratalá Tejada M, et al. Theories and control models and motor learning: clinical applications in neuro-rehabilitation. Rev Neurol 2015;30:32-41.

42 Preissner K. Use of the occupational therapy task-oriented approach to optimize the motor performance of a client with cognitive limitations. Am J Occup Ther 2010;64:727-34.

43 Khandare SS, Singaravelan RM, Khatri SM. Comparison of task specific exercises and mirror therapy to improve upper limb function in subacute stroke patients. J Med Dent Sci 2013;7:5-14.

44 Almhdawi KA, Mathiowetz VG, White M, et al. Efficacy of occupational therapy task-oriented approach in upper extremity post-stroke rehabilitation. Occup Ther Int 2016;23:444-56.

45 Bayón-Calatayud M, Gil-Agudo A, Benavente-Valdepeñas AM, et al. Efficacy of new therapies for upper limb neurorehabilitation in stroke patients. Rehabilitación 2014;48:232-40.

46 Michaelsen SM, Dannenbaum R, Levin MF. Task-specific training with trunk restraint on arm recovery in stroke: randomized control trial. Stroke 2006;37:186-92.

47 Pollock A, Farmer SE, Brady MC, et al. Interventions for improving upper limb function after stroke. Cochrane Database Syst Rev 2014:2014:CD010820.

48 Vandana PM, Mohanty P. Effectiveness of mirror therapy in rehabilitation of hand function in sub-acute stroke. Palliat Med Care 2017;4:1-9.

49 Ojeda N, Del Pino R, Ibarretxe-Bilbao N, et al. [Montreal cognitive assessment test: normalization and standardization for Spanish population]. Rev Neurol 2016;63:488-96.

50 French B, Thomas LH, Coupe J, et al. Repetitive task training for improving functional ability after stroke. Cochrane Database Syst Rev 2016;11:CD006073.

51 Pollock A, Baer G, Campbell P, et al. Physical rehabilitation approaches for the recovery of function and mobility following stroke. Cochrane Database Syst Rev 2014;15:CD001920.
52 Arya KN, Pandian S, Kumar D, et al. Task-Based mirror therapy augmenting motor recovery in poststroke hemiparesis: a randomized controlled trial. J Stroke Cerebrovasc Dis 2015;24:1738-48.

53 Sallés L, Gironès X, Martín-Casas $\mathrm{P}$, et al. A neurocognitive approach to recovery of movement following stroke. Phys Ther Rev 2015;20:283-9.

54 Traversa R, Cicinelli P, Oliveri M, et al. Neurophysiological followup of motor cortical output in stroke patients. Clin Neurophysiol 2000;111:1695-703.

55 Ferrer González BM. Adaptación y validación al español de la escala Fugl-Meyer en el manejo de la rehabilitación de pacientes con ictus [Doctoral thesis]. Universidad de Sevilla, 2016.

56 Doussoulin-Sanhueza A, Rivas-Sanhueza R. Validation and use motor activity log and action research arm scales as tools to assess the function of the paretic upper limb after a stroke in clinic and research. Rev Mex Neurocienc 2014;15:138-46.

57 Mathiowetz V, Volland G, Kashman N, et al. Adult norms for the box and block test of manual dexterity. Am J Occup Ther 1985;39:386-91.

58 Gómez-Soriano J, Cano-de-la-Cuerda R, Muñoz-Hellin E, et al. [Evaluation and quantification of spasticity: a review of the clinical, biomechanical and neurophysiological methods]. Rev Neurol 2012:55:217-26.

59 Fernández-Concepción O, Román-Pastoriza Y, Alvarez-González MA, et al. [The development of a scale to evaluate the quality of life in stroke survivors]. Rev Neurol 2004;39:915-23.

60 Domínguez-Morales MR, Balmaseda-Serrano R, León-Carrión J, et al. Functional recovery of cerebrovascular patients after intesive treatment: preliminary data. Revista Española de Neuropsicología 2012;2:44-61.

61 Jiménez-Caballero PE, López-Espuela F, Portilla-Cuenca JC, et al. [Evaluation of the instrumental activities of daily living following a stroke by means of the Lawton and Brody scale]. Rev Neurol 2012;55:337-42.

62 Montaner J, Alvarez-Sabín J. [NIH stroke scale and its adaptation to Spanish]. Neurologia 2006;21:192-202.

63 Arya KN, Verma R, Garg RK, et al. Meaningful task-specific training (MTST) for stroke rehabilitation: a randomized controlled trial. Top Stroke Rehabil 2012;19:193-211.

64 Lisalde-Rodríguez ME, Garcia-Fernández JA. [Mirror therapy in hemiplegic patient]. Rev Neurol 2016;62:28-36. 